\title{
Plasmacytoid Variant of Urothelial Carcinoma in Ureter
}

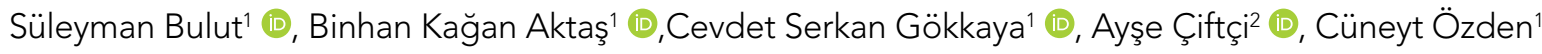 \\ ${ }^{1}$ Department of Urology, Health Sciences University Ankara Numune Training and Research Hospital, Antalya, Turkey \\ 2Department of Pathology, Health Sciences University Ankara Numune Training and Research Hospital, Antalya, Turkey
}

Cite this article as: Bulut S, Aktaş BK, Gökkaya CS, Çiftçi A, Özden C. Plasmacytoid Variant of Urothelial Carcinoma in Ureter. JAREM 2018; 8(2): 112-5.

\begin{abstract}
Plasmacytoid urothelial carcinoma is a relatively rare form of urothelial transitional cell carcinoma, which primarily presents in the bladder. A cautionary approach is required by the pathologist for the final histologic diagnosis. Since the diagnosis is usually challenging, most cases are diagnosed as metastases in the advanced stage. No clear protocol exists in the urology guidelines as a treatment because there are few data present regarding the disease. In the present study, we aimed to present a case of ureteral plasmacytoid variant of urothelial carcinoma, which to our knowledge is the first to appear in the literature.
\end{abstract}

Keywords: Plasmacytoid urothelial carcinoma, ureter, metastasis

ORCID IDs of the authors: S.B. 0000-0002-7607-129X; B.K.A. 0000-0003-0707-9308; C.S.G. 0000-0002-1466-6490; A.Ç. 0000-0001-7524-0912; C.Ö. 00000003-0101-6904.

\section{INTRODUCTION}

Plasmacytoid urothelial carcinoma (PUC) is a very rare form of urothelial transitional cell carcinoma. It is mostly observed in the bladder and is histologically diagnosed by the careful approach of the pathologist. Because it is a highly aggressive disease, there is a lot of work in the diagnosis stage of the urologist and pathologist. According to the World Health Organization's 2004 classification, PUC is defined as one of the rarest types of urothelial carcinoma (1). As the diagnosis is difficult, most of the cases are metastasized at advanced stage. In this study, we aim to present a case of PUC that will be included in the literature for the first time.

\section{CASE PRESENTATION}

A 46-year-old female patient was admitted to our clinic because of left side pain for two months. Computed tomography revealed the following: grade 1-2 level hydronephrosis in the left kidney collector system, dilatation in the middle and proximal section of the left ureter as well as soft tissue contrast that fills the lumen at a distance of 6-7 cm to the ureterovesical junction. The ureter was dilated and tortuous at the proximal of this level. In the distal, there was no dilatation in the ureter. No pathology was observed in the bladder.

Based on this result, the patient was hospitalized for further investigation and treatment. After the necessary routine preparations were completed, the patient was operated under general anesthesia. The bladder was normal in cystoscopy. Then, left ureterorenoscopy was started. However, the ureter could not be crossed upward from the lower one-third segment. It was decided to perform exploration with a left ureter catheter. The patient was turned to the supine position and subsequently explored with the left Gibson incision. In the exploration, the ureter was highly adhering to the surrounding tissue at the levels where the iliac vessels crossed. The ureter was dissected up to the bladder. Mass was observed in the $1 \mathrm{~cm}$ segment in the ureter at the iliac cross-level. Proximal dilatation was observed. The ureter catheter was removed. A 1-cm long incision was made from proximal to ureter, and catheter was delivered to the bladder. It was observed that the catheter was transferred to the bladder, and then frozen was taken from the inflammatory appearance hardness in the ureter. Accordingly, pathological examination revealed an inflammatory and plasmacytoidal reaction, but it could not differentiate between benign or malignant. Therefore, that preparation was examined as a paraffin block. Conversely, we decided to await the pathology result for further treatment, and $4,8 \mathrm{~F} 26 \mathrm{~cm}$ double-J catheter was inserted into the left ureter. The ureter was primary sutured, and the operation was terminated.

The patient's consent was not taken because of the retrospective design of the study.

\section{Pathology}

For submitted biopsy samples, the sections stained with H\&E (Figures 1,2) revealed atypical cells in a uniform appearance, which infiltrate fibromuscular tissue with a small hyperchromatic nucleus and eosinophilic cytoplasm and plasmacytoid appearance. Therefore, immunohistochemical investigations were conducted. In these cells, widespread expression was observed with PanCK, 34BE12 (Figure 3), CK7, and CD138 (Figure 4, 5). Focal expression was observed with p63 (Figure 6), while no expression was observed with kappa, lambda, CK20, thrombomodulin, LCA, chromogranin A, CD56, and CEA, and consequently the present histomorphological and immunohistochemical findings suggest a malignant epithelial tumor, primarily PUC. 


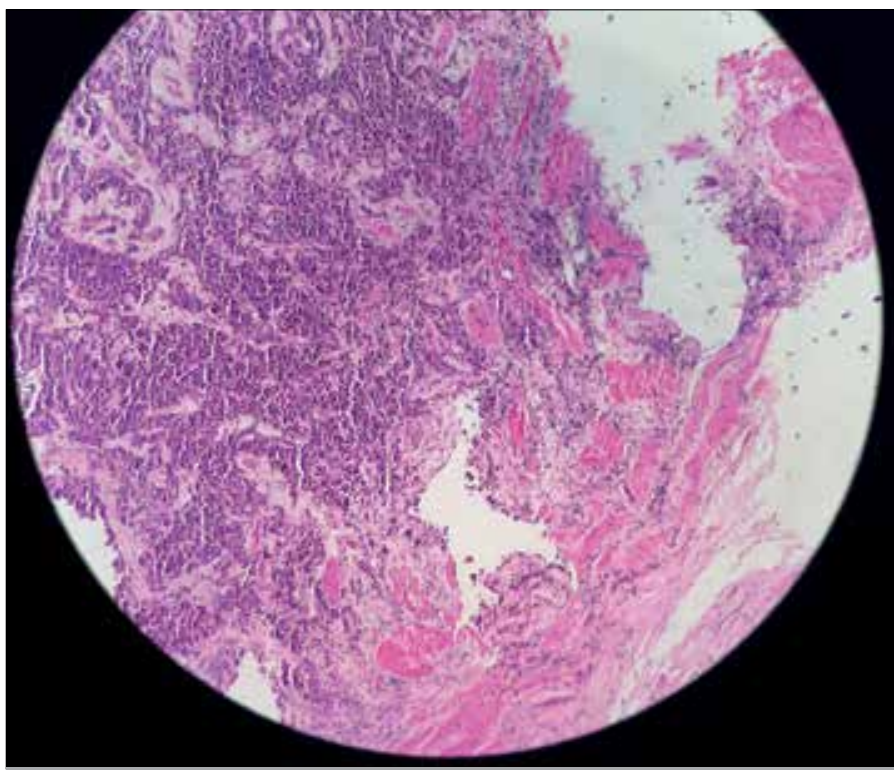

Figure 1. H\&E x 10 BBA: Atypical urothelial cells of uniform and plasmacytoid appearance with narrow eosinophilic cytoplasm with eccentric nucleus infiltrating the muscularis propria

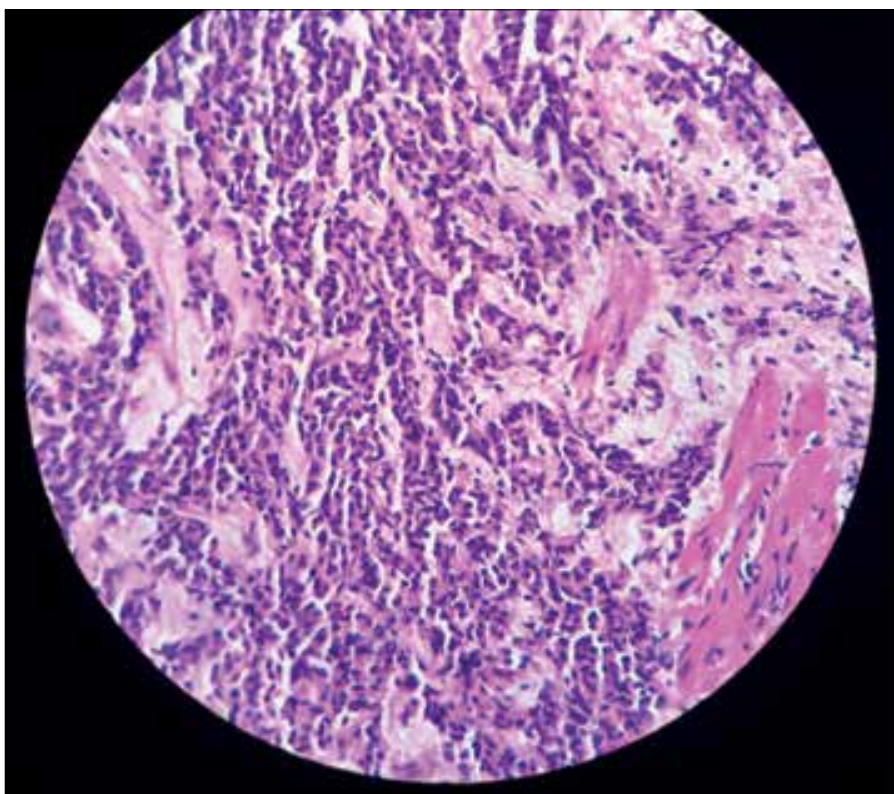

Figure 2. H\&E x 40 BBA: Atypical urothelial cells 145 of uniform and plasmacytoid appearance with narrow 144 eosinophilic cytoplasm with eccentric nucleus infiltrating the muscularis propria 146

This pathology result was reported to the patient, and radical nephroureterectomy and cuff excision from the bladder were planned. The patient was called back to our clinic.

\section{DISCUSSION}

In recent years, with the increasing frequency of diagnosis of urothelial carcinomas, it has started to be seen in serious different variants in patients. Recognition of these lesions is important for their treatment. Because PUC among these variants is observed in advanced age and its diagnosis delayed, its treatment is very difficult, and the disease is aggressive (2).

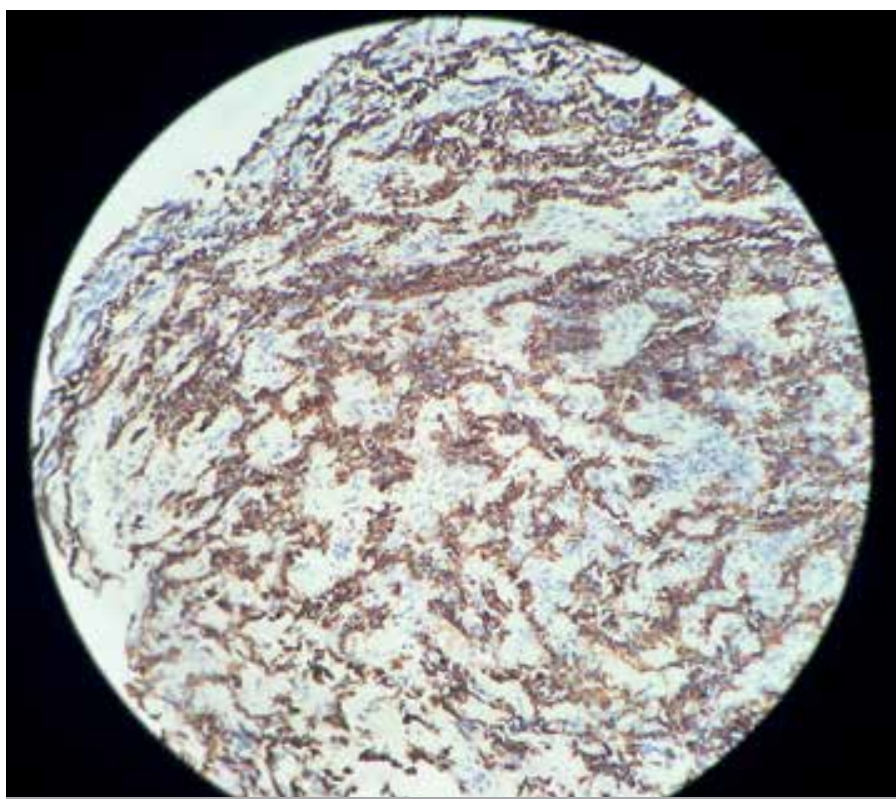

Figure 3. 34BE12 x BBA: Cytoplasmic 147 expression 148 with 34BE12 in plasmacytoid urothelial carcinoma

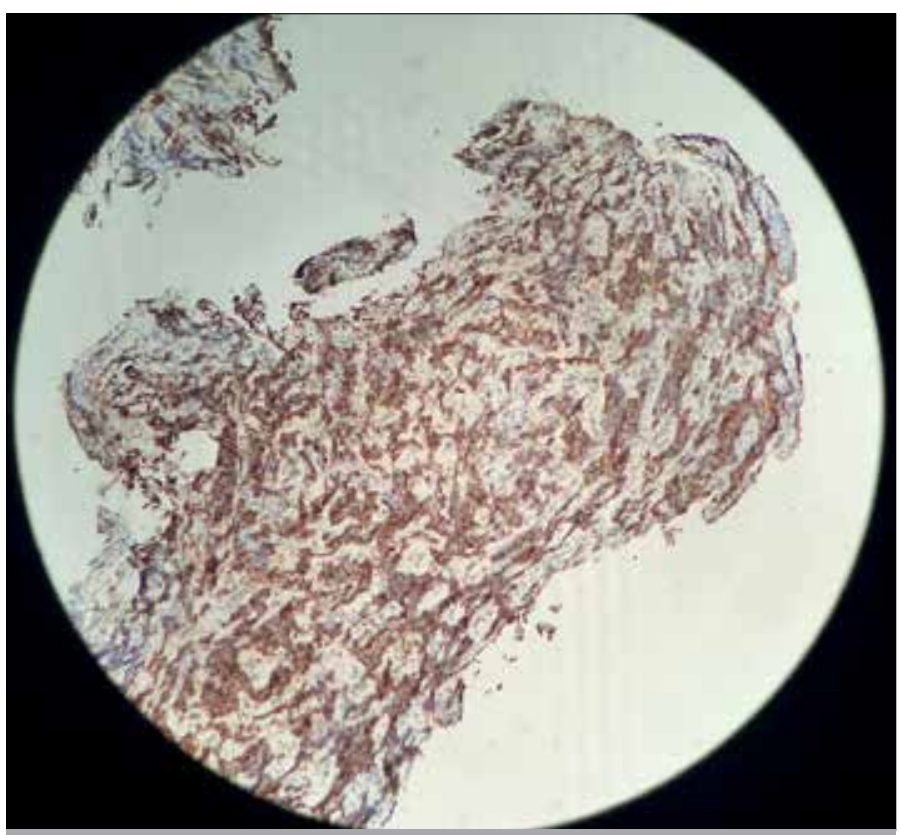

Figure 4. CD $138 \times 10$ BBA: Cytoplasmic and membranous expression with CD138 149

When the literature was reviewed, Sahin et al. (3) presented a transitional cell carcinoma of the bladder containing a plasmacytoid variant in a 63-year-old male patient in 1991. Diagnosis is made after transurethral resection in all patients. However, in our case, the patient's cystoscopy was normal. Moreover, when ureterorenoscopy failed to pass through the ureter because of stricture, the patient was explored, and a diagnosis was made from the tissue sample taken due to the fibrous mass. Because the disease is difficult to diagnose, it can be easily confused with other diseases. These include plasma cell infiltrated cystitis, lymphomas, lymphoepitheliomas, and metastatic carcinomas. Immunohistochemical 


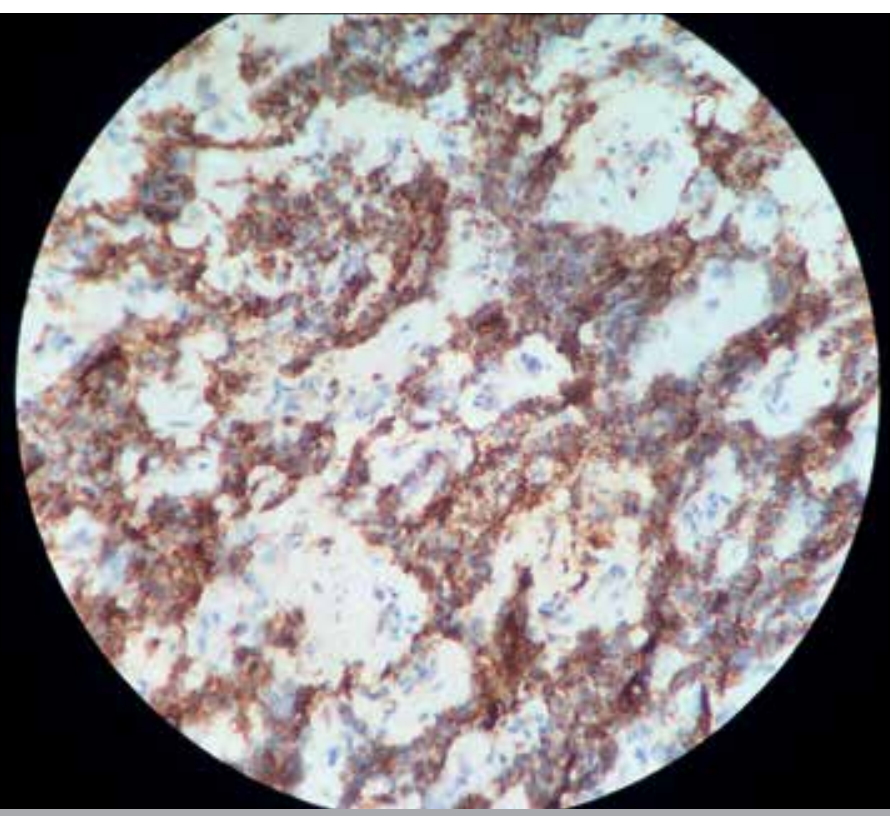

Figure 5. CD $138 \times 20$ BBA: Cytoplasmic and membranous expression with CD138 150

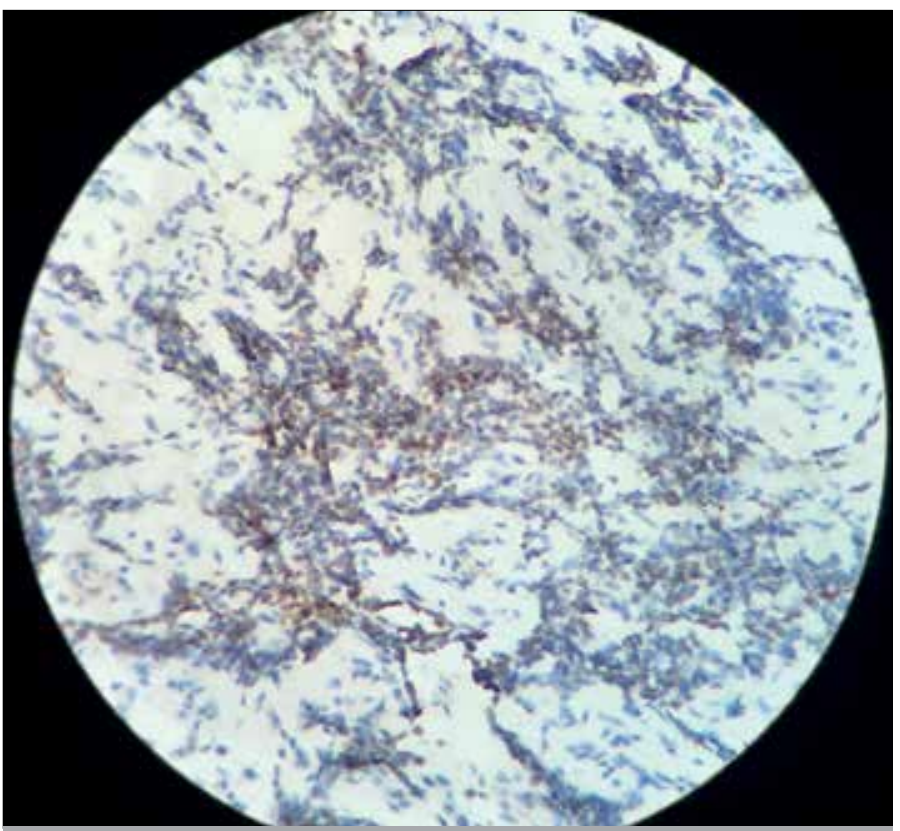

Figure 6. P63 $\times$ 40BBA: Nuclear expression with p63

staining is the most helpful technique in diagnosis. In our patient, the diagnosis was made with immunohistochemical staining.

Apart from the diagnostic difficulties associated with this variant, the clinical, pathological, and immunohistochemical data related to the disease are quite limited (4). The largest series of cases in the literature so far contain 31 and 32 patients $(5,6)$. PUC is estimated to be responsible for approximately $3 \%$ of all muscleinvasive urothelial carcinomas $(5,7)$.

The most common symptoms in patients are hematuria, pollacuria, urgency, and abdominal pain. Because hematuria is not seen until the late stages of the disease, the diagnosis is delayed (8). This delay complicates the treatment of the patient. In our patient, there were no urologic symptoms other than the side pain in the last two months. Because there are few data on the disease, there is no clear protocol in the urology guidelines for treatment. Aggressive therapies are recommended for the treatment of patients with a diagnosis of PUC because the disease tends to be highly invasive. If there is no metastasis after transurethral bladder resection, radical cystectomy should be considered in the first step (8). In addition, both neoadjuvant and adjuvant therapies should be considered in the treatment.

\section{CONCLUSION}

Plasmacytoid urothelial carcinoma is a condition with different pathological and clinical features. Early diagnosis and treatment is very important. Because the diagnosis can be made only after necessary immunohistochemical investigations, there is a lot of work to be done. An aggressive multidisciplinary approach is required in treatment. Therefore, larger data are needed for the disease. In the future, it is expected that there will be different subtypes besides the plasmacytoid variant, and we may say that more work will fall in pathology and urology in the future.

Informed Consent: Due to the retrospective design of the study, informed consent was not taken.

Peer-review: Externally peer-reviewed.

Author Contributions: Concept - C.S.G.; Design - S.B.; Supervision B.K.A.; Resources - S.B.; Data Collection and/or Processing - A.Ç.; Analysis and/or Interpretation - C.Ö.; Literature Search - B.K.A.; Writing Manuscript - S.B.; Critical Review - B.K.A.

Conflict of Interest: The authors have no conflicts of interest to declare.

Financial Disclosure: The authors declared that this study has received no financial support.

\section{REFERENCES}

1. Lopez-Beltran A, Cheng L. Histologic variants of urothelialcarcinoma: differential diagnosis and clinical implications. Hum Pathol 2006; 37: 1371-88. [CrossRef]

2. Zukerberg LR, Harris NL, Young RH. Carcinomas of the urinary bladder simulating malignant lymphoma. A report of five cases. Am $J$ Surg Pathol 1991; 15: 569-76. [CrossRef]

3. Sahin AA, Myhre M, Ro JY, Sneige N, Dekmezian RH, Ayala AG. Plasmacytoid transitional cell carcinoma. Report of a case with initial presentation mimicking multiple myeloma. Acta Cytol 1991; 35 277-80.

4. Rahman K, Menon S, Patil A, Bakshi G, Desai S. A rare case of plasmacytoid urothelial carcinoma of bladder: Diagnostic dilemmas and clinical implications. Indian J Urol 2011; 27: 144-6. [CrossRef]

5. Keck B, Stoehr R, Wach S, Rogler A, Hofstaedter F, Lehmann J, et al. The plasmacytoid carcinoma of the bladder-Rare variant of aggressive urothelial carcinoma. Int J Cancer 2011; 129: 346-54. [CrossRef]

6. Dayyani F, Czerniak BA, Sircar K, Munsell MF, Millikan RE, Dinnery $\mathrm{CP}$, et al. Plasmacytoid urothelial carcinoma, a chemosensitive cancer with poor prognosis and peritoneal carcinomatosis. J Urol 2013; 189: 1656-61. [CrossRef] 
7. Keck B, Wach S, Stoehr R, Kunath F, Bertz S, Lehmann J, et al. Plasmacytoid variant of bladder cancer defines patients with poor prognosis if treated with cystectomy and adjuvant cisplatin-based chemotherapy. BMC Cancer 2013; 13: 71. [CrossRef]
8. Wang Z, Lu T, Du L, Hu Z, Zhuang Q, Li Y, et al. Plasmacytoid urothelial carcinoma of the urinary bladder: A clinical pathological study and literature review. Int J Clin Exp Pathol 2012; 5: 601-8. 